\title{
Multilinguales
}

$14 \mid 2020$

Enseignement universitaire et professionnalisation : enjeux, questionnements et défis socio-économiques

\section{Formation universitaire et insertion professionnelle : une enquête sur l'expérience des diplômés universitaires en langue française au Nigéria}

University education and professional integration: a case study of university French graduates in Nigeria Tajudeen Abodunrin Osunniran et Mohammed Sale Also

\section{(2) OpenEdition \\ Journals}

Édition électronique

URL : https://journals.openedition.org/multilinguales/5465

DOI : $10.4000 /$ multilinguales.5465

ISSN : 2335-1853

Éditeur

Université Abderrahmane Mira - Bejaia

Référence électronique

Tajudeen Abodunrin Osunniran et Mohammed Sale Also, « Formation universitaire et insertion professionnelle : une enquête sur l'expérience des diplômés universitaires en langue française au Nigéria », Multilinguales [En ligne], 14 | 2020, mis en ligne le 10 décembre 2020, consulté le 02 juillet 2021. URL : http://journals.openedition.org/multilinguales/5465; DOI : https://doi.org/10.4000/ multilinguales. 5465

Ce document a été généré automatiquement le 2 juillet 2021.

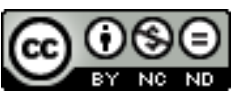

Multilinguales est mise à disposition selon les termes de la Licence Creative Commons Attribution Pas d'Utilisation Commerciale - Pas de Modification 4.0 International 


\title{
Formation universitaire et insertion professionnelle : une enquête sur l'expérience des diplômés universitaires en langue française au Nigéria
}

\author{
University education and professional integration: a case study of university \\ French graduates in Nigeria
}

\author{
Tajudeen Abodunrin Osunniran et Mohammed Sale Also
}

1 Chaque année, les universités, dont l'une des principales responsabilités est de préparer les étudiants à occuper différentes fonctions dans la sphère socio-économique (Tremblay, Groleau \& Doray, 2014), déversent sur le marché du travail des diplômés prêts à être embauchés. Mais une fois sur ce marché, l'un des défis majeurs auxquels font face ces diplômés est l'employabilité, c'est-à-dire leur capacité, de par leurs compétences, à être sollicités sur le marché de l'emploi (Yabouri et al, 2007). En Afrique, l'insertion professionnelle des jeunes diplômés est une question qui continue à faire couler beaucoup d'encre et à susciter beaucoup de recherches, parmi lesquelles Yabouri et al (2007), Boussada \& De Ketele (2008), Ben Halima, Kocoglu \& Ben Halima (2010), Ghouati (2016), Konaté (2017) et Adjegan (2018). Ces recherches se focalisent pour la plupart sur les questions ayant affaire à la performance des diplômés sur le marché du travail, à leurs stratégies et modes d'accès à l'emploi et aux relations qui existent entre la formation, le degré d'insertion professionnelle et le marché du travail. Cette étude se veut une contribution dans le sillage de ces études précédentes. Elle s'intéresse à une filière et à un public particulier dans le contexte nigérian: les diplômés en langue française des universités nigérianes.

Dans ce contexte nigérian, plusieurs chercheurs ont essayé d'exposer les problèmes inhérents à l'enseignement et à l'apprentissage du français dans les universités nigérianes et proposer des solutions visant à les surmonter. À titre d'exemples, Ajiboye 
(1996) a jeté un regard sur la place accordée à l'enseignement de l'oral dans le programme du français universitaire au Nigéria. L'auteur constate que le français oral tel qu'il est conçu dans ce programme est de nature 'autonome, a-situationnel et unidimensionnel' (Ajiboye, 1996: 173) et recommande de ce fait l'adoption d'une perspective interactionnelle de l'enseignement de l'oral qui a 'valeur motivationnelle' et est à même de 'remplir concrètement les fonctions psycho-sociales de la communication' (Ajiboye, 1996 : 173). Onumajuru (2003), pour sa part, s'est penchée sur l'avenir de l'enseignement universitaire du français au Nigéria. Après avoir passé en revue la situation d'alors du français et cerné les problèmes militant contre son épanouissement, elle a suggéré des mesures pour améliorer cette situation et assurer un avenir prometteur à son apprentissage. Simire (2013), quant à elle, a cherché à évaluer le niveau d'un groupe d'étudiants universitaires en immersion au Village Français du Nigéria selon le Cadre européen commun de référence pour les langues. Son étude montre que la majorité de ces enquêtés ont un niveau A1/A1+. Elle invite de ce fait les parties prenantes à prendre en compte cette réalité dans toute révision du programme de formation des étudiants. Okolo (2015), abordant dans le même sens, a examiné les défis que soulèverait l'enseignement du français de base dans les universités nigérianes selon le CECR (Cadre européen commun de référence pour les langues). L'auteure préconise que le modèle d'enseignement prôné par le CECR, outil fiable dans l'enseignement de langues étrangères et pratiqué dans de nombreux pays à travers le monde, pourrait être une solution pour assurer une bonne compétence linguistique de base chez nos étudiants de français. Elle exhorte en conséquence les enseignants à faire preuve de bonne volonté pour relever les défis inhérents à son adaptation.

3 En ce qui concerne les études qui ont trait à cette étude, nous pouvons citer Afolabi (2008), Simire (2008) et Edung et Nyah (2010). La réflexion d'Afolabi (2008), en effet, a porté sur le curriculum en usage dans les départements de français au Nigéria. En identifiant les défauts associés à ce curriculum, il propose des mesures nécessaires en vue de le professionnaliser pour assurer une facile intégration de nos diplômés dans le monde de l'emploi. Dans le même ordre d'idées, l'étude de Simire (2008) a montré comment une meilleure connaissance des réalités du monde de l'entreprise pourrait aider à adapter la formation des apprenants nigérians de français. Elle a abordé, en particulier, le rapport entre le programme de formation et le marché du travail, les perceptions des étudiants sur le marché du travail et le rôle que pourraient jouer les entreprises dans le processus de formation. Enfin, dans leur contribution, Edung et Nyah (2010)se sont interrogés sur la survie des programmes de licence dans nos universités. En observant que ces programmes n'inculquent aux apprenants que des compétences littéraires qui nuisent à leur chance de survie sur le marché du travail, les auteurs recommandent le français de spécialité à travers des programmes de licence à double discipline comme moyen d'assurer la survie de ces programmes et favoriser une meilleure insertion professionnelle des diplômés.

De cette brève revue de littérature sur les recherches relatives à l'enseignement du français dans les universités nigérianes, il y ressort que la question du devenir de nos diplômés sur le marché du travail, abordée dans cette étude, n'est pas nouvelle. Certains s'y sont intéressés avant nous. Cependant, de l'éventail de recherches qui existent dans ce sens, aucune étude, à notre connaissance, n'a cherché à effectuer une enquête de terrain auprès de 'produits' de ces universités sur le marché du travail, surtout en ce qui concerne les réalités auxquelles ils font face et les perceptions qu'ils 
ont de la formation reçue au regard de leur insertion professionnelle. La présente étude cherche à combler ce déficit. Ainsi, se décide-t-elle à aller à la rencontre d'anciens étudiants diplômés et insérés dans le monde du travail pour leur donner la parole afin de partager leurs points de vue sur le système qui les a formés et au moyen duquel ils s'épanouissent et contribuent leur quota au développement socio-économique de leur pays. Ce faisant, elle cherchera à apporter des éléments de réponses aux questions de recherche suivantes :

- Quel est le profil professionnel des diplômés en langue française au Nigéria?

- Quels sont les secteurs où leurs services sont le plus demandés?

- Comment s'est faite leur insertion professionnelle?

- Quelles sont leurs perceptions vis-à-vis des contenus du programme de français des universités?

- Comment pensent-ils que le programme de français peut être révisé afin de rendre les diplômés plus compétitifs sur le marché du travail ?

Mener une étude de ce genre, selon Lamoure (1995, Chap. 1, p. 1), c'est interroger les relations qui existent entre la formation et l'emploi des jeunes et cela permet de mettre en évidence l'écart qui existe entre les acquis universitaires et les exigences du marché du travail. Recueillir le point de vue des diplômés, au regard des défis rencontrés face à leur insertion professionnelle dans notre contexte, pourrait être un indicateur précieux dans la tentative de redynamiser le programme de formation au niveau de l'enseignement supérieur. Par conséquent, cette étude a le mérite de nous fournir des indices qui sont à même de nous aider à réfléchir sur le bien-fondé ou l'utilité des formations que nous dispensons dans nos départements de français.

Comme plan de travail, nous allons tout d'abord donner un aperçu de la filière d'études en français dans les universités nigérianes, ensuite présenter notre méthodologie de recherche avant de passer à l'analyse et à la discussion de nos résultats.

\section{Un aperçu de la filière d'études en français dans les universités nigérianes}

6 Aujourd'hui, le français fait partie des filières d'études dans 49 universités (Jamb Brochure $^{1}$ ) à travers les 170 universités (National University Commission ${ }^{2}$ ) que compte le pays. Cette filière d'études offre des diplomations qui peuvent aller jusqu'à trois niveaux : Licence - Maîtrise - Doctorat. Le premier niveau nous intéresse dans cette étude.

Normalement, le parcours de formation de tout diplôme de licence au Nigéria (excepté quelques filières comme la médecine, la pharmacie, l'ingénierie et l'architecture) est de trois (L2 - L4) ou quatre (L1 - L4) ans : quatre ans pour les élèves ayant réussi au WAEC (diplôme qui s'obtient après 6 ans d'études au secondaire) et trois ans pour les candidats qui possèdent le OND (Ordinary National Diploma) (équivalent du Brevet de Technicien) ou le A'Level (Advanced LevelCertificate) (équivalent du Baccalauréat). Ceux-ci commencent leurs études de licence en L2 (deuxième année de licence). Ainsi, dans le cadre de la licence en langue française, les candidats avec un Baccalauréat francophone ${ }^{3}$ sont admis pour une formation de trois ans.

7 La formation de licence en langue française est sanctionnée par deux diplômes à vocation différente : le B.A. French (licence ès lettres en français) et le B.A. (Edu.) ou B.Ed. 
French (Licence en pédagogie du français). La différence entre les deux réside dans le fait que le deuxième diplôme qualifie au métier de professeur de français dans le secondaire. Il convient de noter, cependant, que ces deux diplômes ne peuvent s'obtenir au sein de la même faculté. Les étudiants en B.A. French sont admis au département de français (ou de langues étrangères ou de langues européennes, selon le cas) de la faculté des lettres alors que les étudiants en B.Ed French sont admis au département de pédagogie des sciences humaines (et sociales) de la faculté des sciences de l'éducation. Ces derniers (B. Ed. French) reçoivent des cours de français ensemble avec leurs homologues en B.A. French dans les départements de français et des cours de pédagogie, dispensés en anglais, dans les départements de pédagogie. Ils n'assistent qu'aux cours de français qui contribuent à leur formation en tant qu'enseignant. C'est donc dire que les étudiants de français en filière générale sont exposés à des cours de français plus variés que ceux en sciences de l'éducation.

Le programme d'études en français offre un enseignement fait de cours magistraux et de travaux dirigés dans des laboratoires de langues équipés de matériaux audio-visuels. En troisième année (L3), ces étudiants - en B.A. et B. Ed. French - doivent passer une année obligatoire d'immersion linguistique au Nigerian French Language Village (NFLV) (Village Français du Nigéria), Lagos, Nigéria ou dans des centres de langues de pays francophones voisins (Bénin, Togo, Tchad, Côte d'Ivoire, Niger) pour ceux qui en ont les moyens. Le NFLV a été établi en 1991 (Afolabi, 2013 : 19) dans la ville de Badagry, située à la frontière entre le Nigéria et le Bénin, pour 'subvenir aux besoins d'acculturation linguistique des étudiants de français dans les universités et les collèges d'éducation nigérians' (Afolabi, 2013: 20) face à l'impossibilité du gouvernement nigérian de continuer d'envoyer ces étudiants pour l'immersion linguistique en France. Ce centre inter-universitaire reçoit la quasi-majorité des étudiants et offre des formations axées sur l'amélioration des compétences communicatives.

\section{Méthodologie}

Cette étude est de nature descriptive et quantitative. Deux types de recherche sont généralement identifiés dans l'étude du rapport entre formation et emploi, à savoir, dans la terminologie anglo-saxonne, les 'follow-up studies' et les 'tracer studies' (Lamoure, 1995: Chap. 2, p.1). Les premières sont des enquêtes de suivi du cheminement scolaire d'étudiants jusqu'à leur insertion professionnelle et les deuxièmes, de nature rétrospective, sont celles menées auprès d'anciens étudiants diplômés et insérés ou pas dans le monde du travail (Béduwé, 2019). Cette étude s'oriente vers la deuxième perspective. Elle se focalise sur des Nigérians, diplômés en langue française (B. A French ou B. Ed. French) dans une université nigériane entre 2007 et 2017 et occupant un emploi soit dans la fonction publique ou dans le secteur privé ou étant installés à leur propre compte sur le sol nigérian. Le choix de 2017 comme date butoir au lieu des dates récentes comme 2018 ou 2019 est de s'assurer que nos enquêtés ont eu le temps de s'insérer dans le monde de l'emploi. En effet, au Nigéria, après la licence, les diplômés doivent passer une année obligatoire de Service National (National Youth Service Corps). Ainsi, en supposant que les diplômés de 2017 aient effectué le service national en 2018, ils ont eu du temps nécessaire pour s'insérer dans le monde du travail. 
10 Les données de cette étude sont collectées à travers un questionnaire en ligne (Google questionnaire). Compte tenu du défi que posait le repérage de nos diplômés dispersés à travers tout le pays et aussi du fait que cette collecte de données s'est déroulée pendant la crise sanitaire de COVID-19, nous avons décidé de recueillir nos données à travers l'échantillon par réseau. Ainsi, un questionnaire en ligne a été élaboré à partir du moteur de recherche Google et le lien fut ensuite disséminé à travers les réseaux sociaux (WhatsApp, Facebook, etc.) d'amis en amis parmi les diplômés considérés dans cette étude. Ce questionnaire est composé de 26 questions faites de questions fermées (17 questions), de questions semi-ouvertes (2 questions) et de questions ouvertes (7 questions). Il est divisé en trois sections. La première section, composée de 8 questions, recense des informations sur la formation du diplômé. Elle cherche à savoir, entre autres, l'université fréquentée, l'année de fin d'études, le diplôme obtenu et les différents autres diplômes obtenus à part la licence en français. Dans la deuxième section, avec 6 questions, l'attention est tournée vers l'expérience professionnelle de l'enquêté. Ces questions concernent le secteur d'activité, la nature de l'emploi, le domaine d'embauche, les années d'expérience, la tâche principale associée à l'emploi et le rôle du français dans l'accomplissement de cette tâche. Composée de 12 questions, la troisième section, enfin, est relative à l'insertion professionnelle. Elle invite l'enquêté à partager son expérience sur son intégration professionnelle en termes de défis rencontrés et de l'adéquation des contenus de formation, et aussi à proférer des solutions en vue d'améliorer le programme d'enseignement.

11 Selon les critères susmentionnés, au sein d'un cadre temporel de deux mois (juin et juillet), 143 diplômés de 25 universités nigérianes ont répondu à notre questionnaire. Le Nigéria est divisé en 6 zones géographiques et nous avons pu avoir des répondants des universités de chaque zone bien qu'il fût difficile, au vu de la méthode de collecte de données, de gérer le nombre venant de chacune de ces différentes zones. Ainsi, nous avons 92 (64\%) répondants du sud-ouest, 19 (13\%) du nord-ouest, 11 (8\%) du sud-est, 08 (6\%) du nord-est, 07 (5\%) du sud-sud et 06 (4\%) du nord-central. Cette inégalité ne porte pas atteinte à la fiabilité de notre étude, car quelle que soit l'université, les curricula des différentes filières sont sensiblement les mêmes sur le territoire nigérian parce qu'ils sont régulés par la Commission Nationale des Universités (National University Commission).

\section{Analyse des données}

Les données collectées ont été téléchargées de Google Responses sur un fichier Excel et les analyses quantitatives ont été effectuées à partir de ce fichier.

\subsection{Le profil professionnel des enquêtés de l'étude}

Les répondants de notre échantillon proviennent, par ordre d'importance, des cohortes de 2017(17\%), 2010(13\%), 2009(11\%), 2012 \& 2015(10\%), 2008(09\%), 2014\& 2016(08\%), 2007\&2013(06\%) et de 2011(02\%).

Tableau 1 : Distribution des répondants en fonction de la cohorte

\begin{tabular}{|l|l|l|} 
Cohorte & Effectif & Pourcentage \\
\hline
\end{tabular} 


\begin{tabular}{|l|l|l|}
\hline 2007 & 08 & $06 \%$ \\
\hline 2008 & 13 & $09 \%$ \\
\hline 2009 & 15 & $11 \%$ \\
\hline 2010 & 19 & $13 \%$ \\
\hline 2011 & 03 & $02 \%$ \\
\hline 2012 & 14 & $10 \%$ \\
\hline 2013 & 08 & $06 \%$ \\
\hline 2014 & 12 & $08 \%$ \\
\hline 2015 & 14 & $10 \%$ \\
\hline 2016 & 12 & $08 \%$ \\
\hline 2017 & 25 & $17 \%$ \\
\hline Total & 143 & $100 \%$ \\
\hline
\end{tabular}

Parmi ces répondants, $71 \%$ ont étudié le B.A. French et $29 \%$, le B.Ed. French. Ainsi, $29 \%$ de nos répondants ont été formés pour l'enseignement et $71 \%$ pour travailler dans les autres secteurs socioéconomiques. Le dépouillement de nos données montre que les répondants de notre échantillon se retrouvent dans divers secteurs de l'économie, mais le secteur de l'éducation, surtout de l'enseignement secondaire, est de loin celui qui absorbe la majorité d'entre eux.

Tableau 2 : Distribution des répondants en fonction des secteurs employeurs

\begin{tabular}{|l|l|l|l|}
\hline & Enseignement & Autres secteurs & Total \\
\hline Répondants avec un B.A. French & $68(67 \%)$ & $33(33 \%)$ & $101(100 \%)$ \\
\hline Répondants avec un B.Ed. French & $39(93 \%)$ & $03(07 \%)$ & $42(100 \%)$ \\
\hline Tous les répondants & $107(75 \%)$ & $36(25 \%)$ & $143(100 \%)$ \\
\hline
\end{tabular}

Ainsi, 107 (75\%) répondants travaillent dans le secteur de l'éducation et 36 (25\%), dans d'autres secteurs. La majorité $(93 \%)$ des diplômés à vocation pédagogique travaille dans l'enseignement et le reste $(07 \%)$, dans le secteur bancaire. Les diplômés formés en filière générale sont également embauchés pour la majorité $(67 \%)$ dans le secteur de l'enseignement et $33 \%$ sont répartis dans d'autres secteurs comme la traduction et l'interprétariat, la banque, le journalisme, la santé, l'armée, la fabrication, le divertissement, les TIC (Technologies de l'Information et de la Communication) et l'assurance. 

publique et $13 \%$ sont installés à leur propre compte. Par ailleurs, la majorité (81\%) de ceux qui travaillent dans les secteurs public et privé sont employés à temps plein contre $19 \%$ employés à temps partiel. Ces enquêtés sont dotés d'une expérience professionnelle qui va en ordre d'importance de 9 ans (22\%), 5 ans (15\%), 7 ans (14\%), 3 ans (12\%), 2 ans (10\%), 8 ans (9\%), 6 ans (7\%), 1 an (6\%) à 4 ans (5\%).

\subsection{L'insertion professionnelle} diplômés en langue française au Nigéria. Les deux grands groupes de diplômés (B.A. French et B.E.d French) sont insérés dans des secteurs qui ne reflètent pas nécessairement le diplôme et la formation reçue. Tout porte à croire que les réalités associées à chaque groupe dans le monde professionnel ne seront pas les mêmes. Ainsi, nous nous attellerons dans les lignes qui vont suivre à voir comment se fait l'insertion de chaque groupe, quels sont les défis rencontrés et quelles suggestions fait chaque groupe au regard des contenus de la formation reçue.

\section{L'insertion des diplômés avec un B.A. French}

16 Cette catégorie représente $71 \%$ des répondants de notre corpus. Ils sont formés à une filière générale. Ils sont donc aptes à être employés dans les différents domaines d'activités socio-économiques du pays où les services d'experts en langue française sont requis. Mais ce que nous remarquons est que la majorité (67\%) d'entre eux est embauchée dans le secteur de l'enseignement, avec un taux de pénétration dans les entreprises relativement faible (33\%).

17 La plupart de ceux qui exercent dans le secteur de l'éducation sont professeurs de français au secondaire; deux sont enseignants dans le supérieur et cinq exercent des fonctions administratives au sein d'établissements secondaires. Dans cette catégorie, $85 \%$ affirment qu'ils utilisent le français dans l'exercice de leur fonction. Malgré le fait qu'ils ne soient pas formés pour enseigner, $65 \%$ de ce groupe jugent avoir eu une insertion facile. A la question de savoir s'ils ont eu besoin de formation supplémentaire pour mieux accomplir leurs tâches professionnelles, $49 \%$ affirment avoir suivi des formations en cours d'emploi afin d'obtenir des diplômes additionnels pour faire partie de la famille des pédagogues. En ce qui concerne les compétences langagières les plus problématiques dans l'exercice de leur fonction, $47 \%$ trouvent que la communication orale posait le plus de défis contre $13 \%$ pour la communication écrite et $16 \%$ pour les deux compétences. Seulement $24 \%$ n'ont pas de difficultés à s'exprimer convenablement à l'oral et à l'écrit. Pour ce qui est de l'adéquation des contenus du programme de formation des Départements de français, $25 \%$ y donnent un jugement favorable, $41 \%$, un jugement défavorable et $34 \%$ y sont indifférents. Ceux qui émettent un jugement favorable estiment que ces programmes permettent de développer des compétences nécessaires pour exercer dans le monde du travail. Ceux qui sont en défaveur, cependant, pensent que ce programme n'est pas en tandem avec les réalités du monde professionnel.

18 Pour les diplômés avec un B.A. French exerçant dans le monde de l'entreprise, 67 \% jugent avoir eu une insertion facile. Mais $70 \%$ affirment qu'ils ont eu besoin d'une 
formation de mise à niveau avant de pouvoir exercer. Quant aux compétences qui leur ont été source de difficultés, la communication orale (37\%) s'est révélée plus problématique. Interrogés sur leur niveau de satisfaction par rapport aux contenus du programme, $51 \%$ émettent un avis défavorable et $19 \%$, un avis favorable. Par ailleurs, $54 \%$ de ces enquêtés affirment avoir obtenu un diplôme professionnel en plus de leur licence en langue française pour faciliter leur professionnalisation. De plus, seulement $33 \%$ affirment utiliser régulièrement le français dans l'exercice de leur fonction et $27 \%$ affirment ne l'utiliser que de temps en temps.

\section{Les diplômés avec un B.Ed. French}

Les diplômés avec un B.Ed. French sont formés à la pédagogie du français. C'est à eux que revient la tâche de dispenser l'enseignement du français aux écoles secondaires du pays. Ils représentent $29 \%$ des répondants de notre corpus. La majorité (93\%) d'entre eux, comme il fallait s'y attendre, sont enseignants. Seulement $7 \%$ exercent dans d'autres secteurs.

Les enseignants pour la majorité sont professeurs de français à l'école secondaire. Certains qui possèdent des diplômes élevés enseignent dans le tertiaire. À $85 \%$, ils affirment utiliser régulièrement le français dans l'exercice de leur métier d'enseignant. Aussi, 49 \% affirment s'être intégrés facilement contre $51 \%$ qui ont eu une intégration difficile. Ils sont $67 \%$ à affirmer qu'ils n'ont pas eu besoin de formation de mise à niveau avant de s'insérer dans la profession. Quant aux compétences problématiques, la communication orale (36\%) est plus problématique, suivie de la communication écrite (31\%) et des deux compétences (10\%). Seuls $44 \%$ affirment être satisfaits du contenu du programme de français, $23 \%$ sont indifférents et $33 \%$ n'en sont pas satisfaits.

Les diplômés à vocation pédagogique de notre corpus qui exercent dans le monde de l'entreprise travaillent dans le secteur bancaire. Ils affirment tous avoir eu besoin d'un diplôme professionnel pour s'insérer dans le monde du travail. De même, 67 \% utilisent le français dans leur métier et ce même pourcentage juge que l'insertion a été facile, mais moyennant une formation de mise à niveau. La communication écrite s'est révélée plus problématique $(67 \%)$ et dans l'ensemble, ils émettent un avis favorable (67\%) quant aux contenus du programme de formation.

\section{Discussion}

21 Nos questions de départ ont été formulées dans le but d'avoir des indices sur la réalité professionnelle de nos diplômés sur le marché du travail nigérian.

L'enseignement reste le débouché dominant disponible pour les diplômés de notre échantillon. En nous fiant à notre connaissance du contexte, nous pouvons affirmer que c'est une réalité qui se confirme sur le terrain. C'est aussi une réalité qui n'est cachée de personne. Si elle se justifie pour les diplômés en pédagogie, qu'en est-il de ceux qui ont suivi une filière générale ? Il y a une décennie, Edung et Nyah observaient que :

...les futurs étudiants se détournent du programme de B.A. (French) parce que le seul métier assuré par les seules compétences qu'ils acquièrent dans ce programme, tel que cela se fait actuellement, c'est l'enseignement, considéré comme dérisoire dans notre société, il s'ensuit qu'il n'y a pas assez de professeurs compétents dans nos cours secondaires. (Edung et Nyah, $2010: 62$ ) 
le terrain, bien que le nombre d'étudiants qui s'intéresse au français ait considérablement évolué au cours des années, l'enseignement, un métier que beaucoup dédaignent à cause de ses conditions de travail déplorables, reste le débouché le plus facilement disponible. Ce désenchantement ressort des commentaires de certains de nos enquêtés :

« French students are subjected to become French teacher. I am not going to be surprised if $95 \%$ of the graduated students are all teachers. » (Réponse 28), « French should not be taught to become a teacher.» (Réponse 53), «Jobs for French graduates should not be limited to teaching in the classroom " (Réponse 115).

Deux facteurs principaux justifient, à notre avis, cet état de fait: l'insuffisance de professeurs de français et la difficulté de nos diplômés à accéder facilement aux secteurs du monde de l'entreprise. En effet, les diplômés en licence pédagogique ne sont pas suffisants pour satisfaire la demande en enseignants sur le territoire. Ce déficit fait qu'il existe beaucoup d'établissements scolaires (primaires et secondaires) où le français n'est pas enseigné au Nigéria, bien que le gouvernement ait déclaré son enseignement obligatoire de la quatrième année du primaire jusqu'à la troisième année $\mathrm{du}$ premier cycle du secondaire et facultatif au second cycle (National Policy on Education, 1998). Ce manque à combler fait que toute personne intéressée à l'enseignement est embauchée d'office sans aucun égard à la nature de son diplôme. Aussi, faute de se trouver un emploi en relation avec la formation reçue, les diplômés en licence générale se replient, malgré eux, immédiatement sur l'enseignement. Par conséquent, comme l'ont remarqué Edung et Nyah, cités plus haut, ce secteur est peuplé de professeurs de français non-formés à la tâche, mais aussi non-motivés. Le secteur privé de l'enseignement absorbe la plupart de ces diplômés, car c'est dans les écoles privées que s'apprend en majorité le français au Nigéria. Pour certaines de ces écoles, l'apprentissage du français sert d'appât pour attirer de la clientèle, car la présence du français parmi leurs matières scolaires permet de courtiser les parents qui désirent offrir à leurs enfants la maîtrise d'une autre langue étrangère à part l'anglais.

Dans l'ensemble, $60 \%$ de nos enquêtés estiment qu'ils ont pu facilement s'intégrer dans le marché du travail. Ce qui retient notre attention, cependant, est le fait que dans le secteur de l'éducation, le degré d'insertion des diplômés qui ont suivi une filière générale est meilleur (67\%) par rapport aux ayant-droits de ce métier qui ont une facilité d'insertion de $49 \%$. Cela s'explique sans doute par la concurrence que subit ce deuxième groupe du premier et du fait que, comme remarqué précédemment, la nature $\mathrm{du}$ diplôme semble jouer un rôle secondaire dans l'employabilité de ce secteur. Il semble d'ailleurs que les employeurs préfèrent accorder plus d'importance à la compétence communicative qu'à la compétence pédagogique. Un diplômé avec un B.A. French qui s'exprime bien en français a plus de chance d'être embauché comme enseignant qu'un diplômé formé à l'enseignement mais qui peine pour s'exprimer convenablement en français. Pour les diplômés exerçant dans le secteur de l'entreprise, la majorité (67\%) affirme aussi avoir eu une insertion facile, mais moyennant une formation supplémentaire ou de mise à niveau (pour $69 \%$ d'entre eux). Dans l'ensemble, afin d'améliorer leurs conditions d'insertion professionnelle, soit en affichant une double compétence ou en spécialisant leurs compétences (Béduwé, 2019), 50 \% de nos enquêtés affirment avoir acquis des diplômes additionnels.

25 Par ailleurs, cette étude révèle que bien que nos diplômés soient des experts en langue française, cette dernière ne rentre pas toujours en ligne de compte dans 
l'accomplissement de leurs tâches. Le français, en effet, est une langue étrangère au Nigéria. C'est une langue dont l'usage est limité parce qu'elle ne s'utilise pas, contrairement à l'anglais, langue officielle, dans les activités socio-économiques du pays. Les diplômés en langue française qui sont professeurs enseignent le français. Donc, pour ce groupe, le français est utile dans l'exercice de leur fonction. Mais cela n'est pas toujours le cas pour ceux qui travaillent dans le monde de l'entreprise. Seulement $30 \%$ de nos répondants de ce secteur utilisent régulièrement le français dans leurs lieux de travail, $28 \%$ l'utilisent quelquefois et $42 \%$ affirment ne jamais l'utiliser. Une conséquence que l'on peut tirer de ce résultat, si l'on se permet à des généralisations, est que le contexte anglophone où nos diplômés sont appelés à exercer affecte leur pertinence. Dans un tel environnement, seules les entreprises d'envergure internationale auront besoin de leurs services. À défaut de trouver un emploi dans ces genres d'entreprises, ils acceptent de travailler dans des secteurs où leur statut d'expert en langue n'a aucune importance.

En outre, en termes de compétence communicative, tout n'est pas reluisant pour nos diplômés. Seulement $23 \%$ estiment s'exprimer parfaitement à l'oral et à l'écrit en français ; $41 \%$ ont des difficultés à s'exprimer convenablement à l'oral, $21 \%$ à l'écrit et $15 \%$ à l'oral et à l'écrit. De ce fait, tout laisse à penser qu'à la fin de leurs études, beaucoup de diplômés n'arrivent pas à s'exprimer librement dans la langue qu'ils ont étudiée. Ainsi, l'immersion linguistique qui est censée améliorer la compétence communicative, surtout orale, de nos étudiants n'arrive pas à remplir pleinement ce rôle et beaucoup d'étudiants ont fait écho de ce fait dans leurs commentaires.

Quant à la pertinence des contenus du programme de formation, $42 \%$ des répondants jugent qu'il n'y a pas d'adéquation entre la formation reçue et les réalités du monde travail ; $31 \%$ pensent qu'il y a adéquation et $29 \%$ sont indécis. La majorité de ceux qui émettent un jugement favorable quant à la pertinence de la formation reçue sont ceux qui exercent dans l'enseignement.

De surcroît, cette étude a aussi cherché à savoir le rôle du Français sur Objectifs Spécifiques (FOS) dans la professionnalisation de nos diplômés. Des questions dans ce sens ont cherché à savoir si ce cours est dispensé dans tous les Départements et si elle est utile dans la professionnalisation des enquêtés. C'est un cours introduit dans nos programmes au cours de la dernière révision des contenus du programme de français des universités en tant que module visant à accoutumer nos étudiants à réaliser, avec le français, certaines tâches de différents domaines (secrétariat, hôtellerie, banque, santé, industrie, etc.) auxquelles ils pourraient être confrontés dans le monde professionnel. Il s'offre sur deux semestres en quatrième année de licence (L4). Il se présente alors comme une tentative pour compenser le fait que nos apprenants n'ont pas de compétences autres que langagières pour occuper des postes spécifiques sur le marché du travail. À $62 \%$, nos répondants affirment avoir suivi ce cours et $43 \%$ de ce pourcentage reconnaissent que ce cours a eu un impact positif dans l'exercice de leurs fonctions. Mais le fait que $38 \%$ aient affirmé ne pas avoir suivi ce cours atteste du fait que son enseignement n'est pas encore généralisé dans toutes les universités nigérianes où s'offre le français. 


\section{Suggestions proposées par les diplômés}

28 marché du travail nigérian. Au premier abord, elles attirent notre attention sur le besoin d'adopter de nouvelles postures d'enseignement et d'apprentissage pour améliorer la compétence communicative de nos apprenants. L'apprentissage et l'enseignement $d u$ français au Nigéria a ses tares et c'est, en effet, un secret de Polichinelle que nos apprenants à la sortie des universités ont encore des difficultés à s'exprimer convenablement à l'oral et à l'écrit en français. Si nos diplômés manquent d'une compétence acceptable dans la langue qu'ils ont apprise, ils seront obligés d'aller chercher fortune dans d'autres disciplines parce que personne ne chercherait à employer un diplômé en français qui a du mal à s'exprimer convenablement dans cette langue. Force est de constater que le Village Français du Nigéria dont la tâche est de faciliter l'acculturation linguistique' (Okeke, 2005 :103) des étudiants de français en leur fournissant 'un niveau comparable à celui d'un apprenant ayant séjourné dans un pays francophone' (Simire, 2005 : 79) arrive péniblement à remplir cette obligation. Il est donc temps de revoir sa structuration et son fonctionnement afin de le rendre plus performant.

31 Par ailleurs, il ressort également de l'étude que le secteur de l'éducation regorge de diplômés en français non qualifiés à la tâche. Cela a un impact sur les produits qui sortent de nos écoles secondaires et par ricochet, sur la qualité des candidats qui se présentent pour des études supérieures en français. À défaut de pouvoir contrôler les diplômés qui s'introduisent dans le domaine de l'éducation, suite à la pénurie d'enseignants, il est nécessaire de rendre obligatoire à ceux qui n'ont pas reçu de formation en pédagogie la régularisation de leurs statuts en se professionnalisant par l'obtention du PGDE - un diplôme post-licence en pédagogie qui s'obtient après une année d'étude. Il est bien vrai que, dans la fonction publique, pour obliger cette catégorie de diplômés, toutes disciplines confondues, à régulariser en cours d'emploi 
leur situation professionnelle, on fait de l'obtention de ce diplôme (PGDE) une condition pour l'avancement. Mais dans le secteur privé, où exercent d'ailleurs bon nombre d'enseignants de français, c'est malheureusement le laisser-faire. Nous recommandons donc que ce même critère soit imposé dans ce secteur. Dans le même ordre d'idées, il y a besoin de rendre attractif aux élèves le métier d'enseignant et, dans notre contexte, les encourager à s'inscrire au programme de B.Ed. French où sont le plus souvent déversés, malgré eux, les candidats qui ne remplissent pas les conditions pour une admission au programme de B.A. French.

En outre, il nous incombe de professionnaliser nos programmes afin de diversifier les secteurs d'insertion de nos étudiants. Il faut commencer à mettre l'accent sur l'employabilité de nos diplômés et à tenir compte de possibles 'débouchés professionnels' (Edung et Nyah, 2010: 58) dans les débats sur le devenir de l'enseignement et de l'apprentissage du français au Nigéria. Simire (2012: 407) observait que "[l]es universités nigérianes, en tout cas, dans les départements de français n'ont pas encore trouvé le moyen de relier l'apprentissage du français à des professions susceptibles d'intéresser les apprenants. » Près d'une décennie après cette observation, cette solution idoine se fait toujours attendre. Plus loin, elle remarque que " [s]i le rôle de l'université est d'offrir des formations, elle doit aussi s'assurer que ces formations répondront aux besoins de ceux qui sont susceptibles de les embaucher.» (Simire, 2012 : 407). C'est à notre tour de faire écho de cette nécessité d'allier les savoirs et les compétences développés dans nos départements de français aux besoins requis sur le marché du travail. C'est à travers cela que nous arriverons à donner de l'espoir et une chance à ces diplômés sur un marché du travail en constante mutation. Il est vrai que seules les entreprises d'envergure internationale auront besoin de leurs services, mais leurs capacités d'insertion seront favorisées s'ils peuvent allier à leurs compétences langagières d'autres compétences professionnelles. Sur le terrain, un de nos répondants a émis une remarque qui doit nous interpeller tous, à savoir que : « French aloneis not enough as French graduatescouldeasilybereplaced by French speakers withotherskillssuch as project management, journalism, humanresources, accounting, etc. » (Réponse 38).

33 Ainsi, si on ne prend garde, nos diplômés risquent d'être dispensables, car concurrencés par des diplômés de pays francophones voisins qui viennent chercher fortune dans le pays. Simire (2012: 413) parle de la nécessité d'une 'double compétence' qui permettra à nos apprenants d'avoir une maîtrise de la langue apprise mais aussi une 'bonne compréhension' du domaine (tourisme/hôtellerie, secrétariat, commerce, import/export, relations internationales, etc.) où ils pourront se retrouver. Ce manque à combler dans nos programmes de formation explique pourquoi les entreprises qui recrutent certains de nos enquêtés sont obligées de leur donner des formations de mise à niveau pour optimaliser leur rendement. L'introduction des cours de FOS est louable et nous encourageons tous les départements de français au Nigéria à le rendre plus dynamique afin de contribuer à la professionnalisation de nos étudiants. Pour ce faire, le temps alloué à son enseignement, le caractère idoine des contenus du cours, la compétence des enseignants en la matière sont les facteurs à prendre en compte dans ce processus de dynamisation.

Nous pensons, enfin, qu'il faut également encourager, comme l'ont bien recommandé avant nous Edung et Nyah (2010) et Ojo (2006), le programme de licence à double discipline (B.A. Combined Honours) où les étudiants suivront un double parcours 
d'études: en français et dans une filière professionnelle comme le management, le marketing, la comptabilité, les relations internationales, etc. Cela ouvrira plus d'opportunités à ces diplômés sur le marché du travail en les rendant aptes à un poste exigeant la connaissance soit de la langue française, soit de cette autre discipline ou des deux (Edung et Nyah, 2010:69). Certains départements d'ailleurs ont emprunté cette direction en offrant des licences à double disciple. Mais la seule combinaison en vogue à ce jour est le programme de B.A. (Combined Honours) French/International Relations (licence en Français et Relations Internationales). C'est une bonne ébauche, il ne reste qu'à penser ces genres de programme avec d'autres disciplines scientifiques.

\section{BIBLIOGRAPHIE}

ADJEGAN, K. II, « Etude d'effets du projet « Agir pour l'insertion professionnelle des jeunes au Togo à travers l'offre de services financiers et non financiers adaptés » sur les jeunes entrepreneurs artisans ", Rapport d'étude en Master 2 Etudes du développement - Parcours Expertise économique des politiques et projets de développement (EPOLPRO), Dominante Politiques. IEDES, Université Paris 1, Panthéon Sorbonne, 2018.

AFOLABI, S., « Pour la professionnalisation de la langue en français dans les universités nigérianes ", Revue de l'Association Nigériane des Enseignants Universitaires de Français (RANEUF), vol. 1, No. 5, 2008, pp. 2- 14.

AFOLABI, S., «Le Village Français du Nigeria : bilan de deux décennies d'engagements linguistique et académique », Revue de l'Association Nigériane des Enseignants Universitaires de Français (RANEUF), vol. 1, No. 10, 2013, pp. 19-41.

AJIBOYE, T., «Le français universitaire au Nigéria : Constitution et pédagogisation de l'oral », The West African Journal of Language, Literature\&Criticism (A Multi-lingual Bi-Annual), vol. 1, No. 1, 1996, pp. 160-174.

BÉDUWÉ, C., « Préface : La valeur professionnelle des parcours de formation », dans CALMAND, J., LEMISTRE, Ph. (coord(s)), Céreq Echanges, Effet du parcours à diplôme donné sur l'insertion professionnelle, Groupe de travail sur l'enseignement supérieur (GTES), 11, 2019, pp. 5-21.

BEN HALIMA, M., KOCOGLU, Y., BEN HALIMA, B., « Insertion professionnelle des diplômés universitaires en Tunisie : Comparaison public-privé », 2010, Disponible sur : https:// hal.archives-ouvertes.fr/hal-00943982. Consulté le 19/06/20.

BOUSSADA, H., DE KETELE, J-M., «L'évaluation de la qualité de la formation et du système d'évaluation universitaire : Le point de vue des diplômés ", Avaliação, Campinas ; Sorocaba, SP, vol. 13, No. 1, 2008, p. 39-61.

EDUNG, M.T.U., NYAH, P., « Le français de spécialité : garant de la survie du programme de "Bachelor's Degree" en français au Nigéria et comment s'y prendre », La Revue des études francophones de Calabar (Calabar Journal of Francophone Studies), vol. 9, No. 1, 2010, pp. 56-76.

FEDERAL REPUBLIC OF NIGERIA (FRN). National Policy on Education. Lagos: Nigerian Educational Research and Development Council (NERDC), 6th ed., 2014. 
GHOUATI, A., « L'insertion professionnelle des diplômés au Maghreb. Quel(s) effet(s) de la professionnalisation des formations?», Communication au colloque international :

Professionnalisation des formations, employabilité et insertion des diplômés, Clermont-Ferrand, Ecole Universitaire de Management, Université d'Auvergne, 2016.

JAMB ADMISSIONS AND MATRICULATION BOARD (JAMB). Jamb Brochure. Disponible sur : https://ibass.jamb.gov.ng/brochure/ programmes Consulté le 18/08/20

KONATE, M. K., «L'insertion professionnelle des diplômés de l'enseignement supérieur au Mali. Enquête auprès des employeurs, des enseignants et du personnel administratif des Instituts d'Enseignement Supérieur ", Projet d'Appui au Développement de l'Enseignement Supérieur (PADES), Consortium de recherche, Marikani, Mali, 2017.

LAMOURE, J., Matériels de formation de l'IIPE. Les études d'insertion professionnelle. Une série de douze modules de formation destinés aux planificateurs et administrateurs de l'enseignement, UNESCO, Institut international de planification de l'éducation, 1995.

NATIONAL UNIVERSITY COMMISSION (NUC). Nigerian Universities. Disponible sur : https:// www.nuc.edu.ng/ Consulté le 18/08/20

OJO, S.A., « Venez parler français avec moi: Let us all appropriate the blessings of the French language », Inaugural Lecture Series, 2006, Lagos: Unilag Press.

OKEKE, V.O., « Les différentes étapes de la dégradation des conditions de l'apprentissage du français au Nigéria et les conséquences inéluctables ", Revue de l'Association Nigériane des Enseignants Universitaires de Français (RANEUF), vol. 1, No. 2, 2005, pp. 96-108.

OKOLO, C. J., « L'enseignement du français de base dans les universités nigérianes : le défi du CECR », Revue de l'Association Nigériane des Enseignants Universitaires de Français (RANEUF), vol. 1, No. 13, 2015, pp. 148-168.

ONUMAJURU, E. M., « L'enseignement universitaire du français au Nigéria : quel avenir ? », La Revue Nigériane d'Études Françaises, vol. 1, No. 8, 2003, pp. 32-42.

SIMIRE, A. B., « Le FOS et son importance dans un centre d'immersion : le cas du Village Français du Nigeria », Revue de l'Association Nigériane des Enseignants Universitaires de Français (RANEUF), vol. 1, No. 2, 2005, pp. 72-95.

SIMIRE, A. B., « Connaître l'entreprise pour mieux adapter la formation des apprenants nigérians de français au monde du travail », Revue de l'Association Nigériane des Enseignants Universitaires de Français (RANEUF), vol. 1, No. 5, 2008, pp. 15-31.

SIMIRE, A. B., « L'enseignement du FOS : quelles perspectives pour le Nigéria ? », dans BARIKI, I. et al (Ed(s).) : Aspects of Language Variation, Acquisition and Use. Festschrift For Prof. E. N. Kwofie, University of Cape Coast, Ghana, The University of Cape Coast Press, 2012, pp. 405-415.

SIMIRE, A. B., « Une évaluation du niveau des étudiants universitaires nigérianes selon le cadre européen commun de référence pour les langues ", dans NWABUEZE, J., et al. (Ed(s)), French Language in Nigeria: Essays in Honour of UFTAN Pacesetters, Benin City: UFTAN Mindex Publishing Co. Ltd, 2013, pp. 49-54.

TREMBLAY, E., GROLEAU, A., DORAY, P., « La professionnalisation des formations universitaires au Québec : Analyse de la planification et de l'évaluation de l'offre de formation dans deux établissements montréalais ", Centre Interuniversitaire de Recherche sur la Science et la Technologie (CIRST), L'Observatoire régional montréalais sur l'enseignement supérieur (ORMES), 2014. 
YABOURI, N., «Formation universitaire et degré d'insertion socioprofessionnelle en Afrique de l'Ouest : Cas des diplômés des filières scientifiques et techniques de l'Université de Lomé au Togo ", Recherche financée par le Réseau Ouest et Centre Africain de Recherche en Education (ROCARE), avec le soutien du projet Centre d'Excellence Régionale UEMOA et du Ministère des Affaires Etrangères des Pays Bas, 2007.

\section{NOTES}

1. ${ }^{1}$ https://ibass.jamb.gov.ng/ brochure/programmes

2. ${ }^{2}$ https://www.nuc.edu.ng/

3. ${ }^{3}$ Le système accepte aussi des élèves ayant fait des études secondaires dans des pays francophones.

\section{RÉSUMÉS}

Cette étude s'intéresse à la situation professionnelle des diplômés en langue française au Nigéria. Elle explore, entre autres, les principaux secteurs pourvoyeurs d'emploi à ces diplômés, les défis qu'ils rencontrent dans le processus d'insertion et les perceptions qu'ils ont de l'adéquation entre la formation dispensée dans les départements de français et les besoins d'un monde du travail anglophone. Les données de l'étude ont été recueillies par questionnaire sur un échantillon de 143 diplômés issus de cohortes d'entre 2007 et 2017. Les résultats de l'étude montrent qu'il y a une faible pénétration des diplômés en langue française dans le monde de l'entreprise. Ces diplômés, eu égard à la formation reçue, semblent plus aptes à trouver du travail dans l'enseignement. Ils sont dans l'ensemble unanimes pour une réforme du programme de français afin d'élargir la gamme d'opportunités auxquelles ils peuvent accéder sur le marché du travail.

This study examines the professional situation of French language graduates in Nigeria. It explores, among other things, the main sectors providing employment to these graduates, the challenges they face in the process of integration and their perceptions of the adequacy between the training provided in departments of French across Nigerian Universities and the needs of an Anglophone labour market. The data of the study was collected through a questionnaire from 143 respondents who graduated between 2007 and 2017. The results of the study show that there is a low penetration of French language graduates into the business world. These graduates, given the training received, seem more likely to find work in education. They unanimously support a reform of the French program in order to widen the range of opportunities they can have access to in the labour market.

\section{INDEX}

Mots-clés : insertion professionnelle, diplômés en langue française, adéquation, formation, emploi

Keywords : professional integration, French languagegraduates, adequacy, training, employment 


\section{AUTEURS}

\section{TAJUDEEN ABODUNRIN OSUNNIRAN}

Department of Foreign Languages, Obafemi Awolowo University, Ile-Ife, Nigeria

MOHAMMED SALE ALSO

Department of Linguistics and Foreign Languages, Bayero University, Kano, Nigeria 